\title{
Rente und subnationale Gewalt. Der Beitrag der politischen Ökonomie
}

\author{
Hartmut Elsenhans
}

\begin{abstract}
Rents are a basic element of the political economy of underdeveloped economies. They hinder and often block the mechanism of social integration through gainful employment and veto the power of labour, which characterizes capitalist societies and the constitution of citizenship. The impact of rent on political structures is, however, ambiguous. Anomie is only one possible result. Hence the link between raw material exports and non-state violence is also ambiguous. Many societies, which are characterized by rents, have developed quite powerful mechanisms of keeping internal peace, possibly with limited participation. The conditions of differential impacts of rent on social structures and political behaviour call for an analysis of internal interest mediation. This research is, however, blocked by an ideological commitment of turf-defending German mainstream constructivism in international relations against the consideration of the political implications of rent because of its dual opposition against political economy and sociological realism in the behaviour of social groups.
\end{abstract}

Keywords: development; rent; counterinsurgency; guerrilla; constructivism

Vom zivilisatorischen Prozess geläuterte Kräfte aus Friedensforschung und Entwicklungsökonomie insbesondere der westlichen Welt suchen Ansprechpartner in einer wachsenden Zahl von gescheiterten Staaten (Failed States) für verbindliche Festlegungen auf westliche (humanitäre) Forderungen. Sie haben mit Hilfe einer sehr kursorischen Politikökonomie die Rente entdeckt. Schon früher hatte man die Finanzierung subnationaler Gewaltakteure aus Drogenanbau und Drogenhandel beobachtet. Fast alle Denunziation der subnationalen Gewaltakteure lässt sich in den Stellungnahmen der Theoretiker der konterrevolutionären Kriegsführung in der Zeit der Entkolonialisierung nachweisen, insbesondere das Interesse der Gewaltakteure an Selbstbereicherung. Nunmehr sieht man in Differentialrenten aus mineralischen Rohstoffen, für die es in bestimmten Ländern des Südens besonders günstige Abbaubedingungen gibt, eine finanzielle Grundlage von Organisationen, die als subnationale Akteure das staatliche Gewaltmonopol in Gebieten schwacher Staatlichkeit in Frage stellen. Die Rente, wirtschaftliche Basis moderner Warlords, erscheint in einer nunmehr wirtschaftswissenschaftlich argumentierenden friedenstheoretischen Literatur als wesentlicher Beitrag der politischen Ökonomie (Kaldor 1999, 102-106; Münkler 2002, 171 ff.; Collier/Hoeffler 2002; Schaffar 2008, 51).

Die Resultate dieser empirischen Studien sind nicht ganz klar, je nach Auswahl des Panels. Die einen entdecken einen Zusammenhang zwischen Renten und Gewalt (Collier 2000; Hugon 2003), die anderen nur zwischen bestimmten Renten und Gewalt (LeBillon 2001; Bulte 2005, 1031; Lujala/Gleditsch/Gilmore 2005), wieder andere sind unklar (Ron 
2005; Humphrey 2005; Fearon 2005; Malešević 2008). Werden alle kleinen Länder der drei Kontinente des Südens einbezogen, ist der Zusammenhang im Regelfall schwach und in Lateinamerika oder auch in Asien eher weniger ausgeprägt als im subsaharischen Afrika. Umfassen die Samples alle Rentierstaaten oder alle Staaten mit hohen Renten, insbesondere die ölexportierenden Länder, ist der Zusammenhang auch schwach. In einigen Ländern scheiterten subnationale Gewaltakteure gerade, weil die anerkannten Regierungen ihnen den Zugang zu Renten verweigerten (Algerien). In anderen Fällen entstanden subnationale Gewaltakteure ohne Zugriff auf externe Renten, verschafften sich aber durch wirtschaftliche Entscheidungen solche Renten, um ihre Bewegungen dann finanziell am Leben zu erhalten (Matthies 2005, 37-42; Chojnacki 2005; Cramer 2002). In anderen Weltgegenden, z. B. in Teilen Indiens, bleiben die Aktivitäten subnationaler Gewaltakteure endemisch, auch wenn sich diese weder schon bestehender Renten abwerfender Produktionen bemächtigen, noch neue Renten generierende Produktionszweige aufbauen können.

Die widersprüchlichen empirischen Befunde sind nur sekundär Folge unzureichender Modellspezifikationen. Sie sind das Ergebnis von Zwängen, die sich aus den Prinzipien von Modellierungen ergeben und die Leijonhuvdud in einer Kritik der Entwicklungstendenzen der makroökonomischen Forschung angegriffen hat: „More and more simpleminded plots but ever more mind-boggling special effects. One would like to look forward for a macroeconomics whose plots will give more insight in the human condition." (Leijonhuvdud 1998, 215)

\section{Der Teil und das Ganze: Das Problem der Einbettung von Rente}

Die empirischen Befunde verweisen auf die Komplexität eines Zusammenhangs zwischen Rente, politischen Strukturen der Interessenvermittlung, Konstitution subnationaler Akteure aufgrund möglicher Interessenaggregationen und daraus abzuleitenden - hier gewaltsamen - Strategien der Interessendurchsetzung. Zur Behandlung von Komplexität wird durch Simplifizierung bei der Modellspezifikation die quantitative Überprüfbarkeit angestrebt, aber der relevante Zusammenhang so verstellt, dass die Ergebnisse banal oder, wie die Widersprüche zum Bezug zwischen Rente und Gewalt zeigen, hingebogen werden.

Die Verbindung von Einkommensarten, Struktur der Interessenvermittlung und Konstitution subnationaler Akteure und deren Strategien ist eine klassische Frage qualitativer Politikforschung, die dabei eine verstehende Methode der Erklärung mit breiter, aber eben nicht quantitativer Empirie verbindet. Häufig wird diese Empirie wegen fehlender statistischer Überprüfung oder gar Überprüfbarkeit und wegen der interpretativen Definition der Fallmerkmale von der quantitativ vorgehenden Forschung als anekdotisch bezeichnet. Von ihren Anhängern werden solche Verfahren damit gerechtfertigt, dass die entscheidenden Verbindungsschritte, die sie in ihren Argumentationen anführen, im Regelfall schwer quantifizierbar sind.

Der klassische Fall für die Kontextabhängigkeit an sich ähnlicher Befunde war in frühen Perioden politikwissenschaftlicher Einführungen die unterschiedliche Funktion der Ministerverantwortlichkeit in repräsentativen Verfassungen. Anhand der englischen Entwicklung wurde den Grundstudiumsstudenten gezeigt, dass die Verantwortlichkeit der Regierungen gegenüber den Parlamenten der Durchsetzung bürgerlicher, (tendenziell) demokratischer Herrschaft diente. Gleichwohl wurde gerade dieses Instrument in Cha- 
teaubriands (1816) Analyse der konstitutionellen Monarchie zur Begrenzung der Reformen genutzt, die eine im Vergleich zu den Kräften des Ancien Régime gleichwohl eher zu Reformen bereite Restaurationsmonarchie zur Abwehr einer neuen Revolution durchzuführen bereit war. Marx $(1859,9)$ versuchte in seiner Kritik der politischen Ökonomie die unterschiedliche Funktion des „Teils“, z.B. der Rente, als Element eines hegelianisch sich immer weiter entfaltenden Ganzen zu behandeln. Weil damit Befunde, Strukturen und Mechanismen auf immer vielfältigere Weise relativiert und einer empirisch exakten Überprüfung von Wirkungszusammenhängen entzogen werden konnten, öffnete dies der politisch motivierten Etablierung nicht überprüfbarer Großtheorien mit angeblich dialektischen Zusammenhängen Tor und Tür.

Die Forderung nach Identifizierung klarer und abgegrenzter Zusammenhänge war eine gesunde Reaktion. Im Anschluss an die beiden Werturteilsdebatten in der deutschen Sozialwissenschaft (Ferber 1966) lässt sich aber folgern, dass die Definition solcher abgrenzbarer Zusammenhänge vernünftig dann erfolgt, wenn sie die Debatten über auf Totalität zielende und historische Genese einbeziehende Großtheorien reflektiert.

Die friedenstheoretische These des Zusammenhangs zwischen Rente und Gewalt hat das nicht getan, obwohl für Rente, definiert als politisch angeeignetes und damit ökonomisch relativ frei verfügbares Einkommen, im Unterschied zum Profit die relative Offenheit der Verwendungsmöglichkeiten wesentliches Kennzeichen ist (Elsenhans 2009, 6). Der Zusammenhang zwischen Rente und politischem Verhalten von subnationalen Akteuren muss deshalb notwendig in eine Debatte über die Ambivalenz von Renten eingebettet sein, die zu durchaus unterschiedlichen politischen Strukturen führen kann, in deren Folge sowohl die Profile der Akteure als auch die Strategien, die sie für den $\mathrm{Zu}$ gang zur Rente wählen, differieren können. Dazu reicht der Hinweis auf die Vermittlung zwischen Auswirkungen der Rente und einer abstrakten Governance oder Staatskapazität nicht aus (wie bei Collier/Hoeffler 2005; Snyder/Bhavnani 2005; Regan 2005; Dunning 2005).

Ein bloßer Blick auf die hier durchaus präsente (Jensen/Wantchekon 2004) und in der Entwicklungsökonomie heiße Debatte über die Auswirkungen von Rente auf Entwicklung zeigt dies: Hier stehen Staple-Theory (Watkins 1963) des Wachstums durch Rohstoffexporte gegen Resource curse (Sachs 1999) und Niederländische Krankheit (Enders 1983) und zwischen beiden vermittelnd die Forderung nach Verbesserung der Terms-ofTrade zur Erweiterung der Finanzierungsspielräume für staatsgetriebenes Wachstum, weil dies die industrielle Diversifizierung zwar finanziell erleichtert, ohne dafür aber Anreize für private Investitionen zu schaffen (schon Singer 1950, 482).

\section{Die Ambivalenz von Rente für Entwicklung}

Zunehmend wird in der Literatur zu Rente und subnationaler Gewalt auf die Kontextabhängikeit rekurriert, die Vermittlung über den Charakter des Staatsapparats. Leider wird dabei die theoretisch isomorphe und schon sehr viel weiter vorangetriebene Theorie des Zusammenhangs zwischen Rente und wirtschaftlicher Entwicklung überhaupt nicht reflektiert. Es gibt Grenzfälle des Zusammenhangs zwischen Rente und Entwicklung, die sich durch Dominanz der ökonomischen Verursachungsmechanismen auszeichnen. Die Staple-Theorie wirtschaftlichen Wachstums (Bertram 1963; Sid Ahmed 1988) nimmt an, dass hohe Exporterlöse aufgrund von Rohstoffreichtum und daraus resultierender Exporte sich niederschlagen in steigenden Masseneinkommen, ohne dass die dadurch hohen Realeinkommen der Arbeitskräfte zu Wettbewerbsnachteilen gegenüber Regionen mit niedri- 
geren Arbeitskosten führen. Dagegen behaupten die Theorien des Resource curse und der Niederländischen Krankheit, dass hohe Wechselkurse in der Folge von Ressourcenreichtum zu Wettbewerbsnachteilen für die Branchen führen, die für den langfristigen technischen Fortschritt von Bedeutung sind.

Bedeutsamer sind die Theorien, in denen die Akteure aufgrund der Gefahren von Unterentwicklung und Deindustrialisierung die spontan sich aus Renten ergebenden Tendenzen zu steuern versuchen. Dabei spielen drei Aspekte eine Rolle:

- $\quad$ Rente ist Folge eines beschränkten Wettbewerbs, entweder aufgrund von natürlichen Monopolen oder aufgrund von politisch geschaffenen Marktbeschränkungen (z. B. Angebotskontrollen bei Rohstoffen).

- $\quad$ Die Akteure können Renten für Entwicklung nutzen, stoßen dabei aber auf Probleme, die Einsicht und damit Lernen erfordern.

- Renten beeinflussen die politischen Strukturen und wirken damit über die Interessenvermittlung auch auf die Strategien von Akteuren und möglicherweise sogar auf die politischen Strukturen zurück und beschränken damit die Optionen der Akteure.

Weil Rente Folge des beschränkten Wettbewerbs ist, setzt Rente auch wesentliche Elemente der Standardkonfiguration kapitalistischer Wirtschaft außer Kraft, auf denen letztlich die Theorie des demokratischen bzw. des liberalen Friedens beruht. Während in der Standardkonfiguration der Makroökonomie keynesianischer Prägung Profite Folge von Investitionsausgaben sind, kann wirtschaftlicher Überschuss bei eingeschränktem Wettbewerb ohne Zwang zu Investitionsausgaben angeeignet werden, so dass Renten entstehen. Ohne hier auf Kontroversen über die Grundlagen kapitalistischen Wachstums einzugehen, lässt sich hinzufügen, dass Wettbewerbsbeschränkungen politisch zu erwarten sind, wenn die Endnachfrage nicht wächst, weil dann die Investitionsmöglichkeiten für Unternehmer beschränkt bleiben und damit die Realisierungsmöglichkeiten für Profite abnehmen. In Anlehnung an Bortkiewicz (1907, 455-459) lässt sich zeigen, dass die wachsende Nachfrage nicht dauerhaft ausschließlich von der Investitionsgüternachfrage kommen kann, weil damit Kapazitätseffekte verbunden sind, die letztlich eine wachsende Massennachfrage erfordern.

Bei noch geringer technischer Entwicklung und einem hohen Nahrungsmittelanteil am Verbrauch (niedrige Realeinkommen) und einem hohen Anteil einer durch abnehmende Erträge gekennzeichneten Landwirtschaft müssen Renten auftreten. Dies wird im Marginalität-cum-Rente-Modell (Elsenhans 1994; Elsenhans 1995) unterentwickelter Wirtschaften dargestellt. Ein Überschuss von Arbeit (weil ein Teil der Arbeitskräfte weniger als den unabweisbaren Subsistenzlohn produziert) schließt die neoklassische Lohndrift aus. Ein gleichwohl bestehender Überschuss an Produktion kann wegen fehlender Nettoinvestitionen (keine Erweiterung des Marktes für Güter, die mit „kapitalisierbaren“, d. h. als Kapitalbesitz akkumulierbaren Investitionsgütern produziert werden) nicht als Profit angeeignet werden und fällt als Rente den politisch Mächtigen, dem Staat oder substaatlichen Akteuren zu. Unterentwickelte Wirtschaften sind grundsätzlich durch eine weite Verbreitung von Renten gekennzeichnet (Elsenhans 2009, 26-31).

Die Einbindung in das kapitalistische Weltsystem verstärkt diese Struktur: Die Übertragbarkeit technischen Fortschritts aus dem Zentrum und die Nachfrage auf einem dynamisch sich entwickelnden Weltmarkt, z. B. für Rohstoffe, führt sektoral begrenzt zu hohen Deviseneinnahmen (viel höheren als bei alternativen Produktionen für den Binnenmarkt). 
Solche exzessiven Renten sind dann nur manifester Ausdruck der Dominanz von Renten in der unterentwickelten Welt, durch die das Überspringen des kapitalistischen Wachstumsimpulses blockiert oder wenigstens denaturiert wird. Warum werden in einigen Fällen solche Renten tatsächlich für die Finanzierung von Entwicklung und die wirtschaftliche Transformation eingesetzt, in anderen dagegen nicht?

\section{Rente als Basis finanzieller Entwicklung}

Unter dem Eindruck des militärischen Vorsprungs des Westens haben seit dem 19. Jahrhundert nicht-kapitalistische Staatsklassen alter tributärer Produktionsweisen Renten eingesetzt, um durch staatlich geförderte nachholende Industrialisierung eine ökonomische Basis für die militärische Modernisierung zu schaffen.

Erfolgreich war dabei Japan wegen des geringen Umfangs von Renten, der trotz politischer Unabhängigkeit eine rasche Einführung von Marktelementen und die Orientierung am Export arbeitsintensiv hergestellter Industrieprodukte erzwang. Im größeren Teil der nicht-kapitalistischen Welt kam es allerdings zu einem Niedergang eines erheblichen Teils der traditionellen Eliten. Sie wurden abgelöst oder nachhaltig durch Aufsteiger aus breiten gesellschaftlichen Schichten erweitert. Diese wollten eine modernistischtechnokratisch mehr oder weniger umfassende Industrialisierung vorantreiben und machten dabei nach der Weltwirtschaftskrise der 1930er Jahre starke Anleihen aus den kapitalismuskritischen Konzepten des seit 1917 existierenden realen Sozialismus. Weil sie die gesellschaftlichen Veränderungen begrenzten, scheiterten sie bei der Verknüpfung zwischen wirtschaftlichem Wettbewerb und wachsenden Massenmärkten mit der Folge betriebswirtschaftlicher Ineffizienz (hohe Kapitalkoeffizienten, geringes Innovationspotential). Die aus Verschuldung erzwungene Hinwendung zum exportorientierten Wachstum seit dem Ende der 1970er Jahre bei arbeitsintensiven Industrieprodukten war für die Wirtschaften am leichtesten, die durch Agrarreformen und/oder Grüne Revolution landwirtschaftliche Überschüsse produzieren konnten. Durch einen lokalen Überschuss an Lohngütern konnten sie unter Kaufkraftparität abwerten. Weil bei Abwertung (= Subventionierung von im Exportsektor beschäftigten Arbeitskräften aus dem lokal produzierten landwirtschaftlichen Überschuss) Exporterlösverluste auftreten, haben die exportorientierten Länder im Regelfall komplementäre Maßnahmen staatlich gestützter Diversifizierung der Produktion (z. B. Erhöhung des lokalen Produktionsanteils) oder Ausweitung des lokalen Anteils der Investitionsgüter ergriffen, also Renten für Industrialisierung eingesetzt (Elsenhans 2006, 232-237).

Ob Renten zur Überwindung von Unterentwicklung eingesetzt werden, hängt von politischen Interessen ab und damit auch von der politischen Kompetenz der Akteure im Verhältnis zum Umfang der Renten (Japan) oder bezüglich günstiger inkrementaler Bedingungen durch einmal getroffene Entscheidungen (Pfadabhängigkeit: Taiwan konzentrierte sich frühzeitig auf Klein- und Mittelbetriebe, Korea konnte den dadurch entstehenden Nachteil wegen Konzentration auf staatlich geförderte Großbetriebe nur durch hohe Anstrengungen ausgleichen, Yi 1988, 56-76). Außerdem spielen die Größe des auch im Fall von Ungleichheit entstandenen Binnenmarkts (in Folge der Importsubstitution in Indien, China und Brasilien), die aus möglicherweise ganz anderen Gründen erfolgten Veränderungen der Agrarstruktur (Taiwan und Korea hatten politisch motivierte Agrarreformen, Thailand hatte eine „ererbte" relativ egalitäre Bodenverteilung) sowie die Aufrechterhaltung von arbeitsintensiven Landwirtschaften als Puffer der Beschäftigung (Chinas Übergang zu Marktwirtschaft im Unterschied zu Russland) eine Rolle. 
Es gibt also eine große Breite der durch die Verfügbarkeit von Renten ausgelösten Verhaltensmuster von Akteuren, die von Strukturen der Ökonomie, der Politik und den dadurch konstituierten Akteuren sowie den von ihnen wahrgenommenen Möglichkeiten abhängen. Die Nutzung von Renten zum Zweck der Bereicherung zahlenmäßig kleiner Gruppen, wie im Theorem des Zusammenhangs zwischen Renten und subnationaler Gewalt angenommen, ist nur eine der Möglichkeiten.

\section{Kein eindeutiger Zusammenhang zwischen Rente und politischem Verhalten}

Rente strukturiert Gesellschaften zugunsten der Mächtigen. Um Rente zur wirtschaftlichen Transformation einzusetzen, muss sie in einem anderen als dem derzeit schon wettbewerbsfähigen Sektor investiert werden. Eine solche „Kanalisierung“ von Renten ist stets der Marktkontrolle entzogen, muss aber nicht notwendig in den Händen eines Staatsgebildes sein: Nach 1973 stiegen die Preise für das bisher billige Nahostöl so stark, dass die Ölfirmen aus ihren bisherigen teuren Ölressourcen außerhalb der OPEC und teilweise auch aus dem zwar verteuerten, aber noch relativ billigen Öl aus den „befreundeten" Ölländern am Golf Renten verdienten, die sie in massive Programme zur Entwicklung neuer Geschäftsfelder (nicht nur alternativer Energien wie Kohle und Uran, sondern auch Bergbau) einsetzten, im Übrigen mit oft begrenztem späterem Erfolg.

Weil aber Organisationen ohne territoriale Kontrolle bei der Aneignung von Renten nur bedingt erfolgreich sein können (,Extraprofite“ können ihnen vom territorial kompetenten Staat weggesteuert werden), stärkt das Auftreten von Renten im Regelfall Territorialverbände. Sind sie international anerkannt, werden sie Staaten genannt, sonst handelt es sich um „subnationale“ Gewalt. Aus soziologischer Perspektive sind dies jedoch Staaten, weil sie für ihr Gebiet das Gewaltmonopol beanspruchen, ihr Territorium zu kontrollieren suchen und die Bevölkerung als Staatsvolk in Beschlag nehmen.

Bei subnationalen Gewaltakteuren handelt es sich um Akteure, die nicht über international anerkannte Staatlichkeit verfügen, gleichwohl aber soziologisch als Staaten anzusprechen sind. Sie werden möglich, weil das internationale System als ,juridical statehood" Staatlichkeit verleiht, auch wenn die Nutznießer nicht über gesellschaftliche Grundlagen von Staatlichkeit im betreffenden Gebiet verfügen (Jackson/Rosberg 1985). Es handelt sich also um Quasi-Staaten (Jackson 1990). Die Rente schafft für solche Quasi-Staaten materielle Grundlagen.

Eng territorial umgrenzte Kontrollmöglichkeiten geben Zugang zu „Punkt“-Renten aus Rohstofflagern (Bulte 2005; LeBillon 2001), ohne dass die Bevölkerung dauerhaft kontrolliert werden müsste. Aus diesen Ressourcen lassen sich viele Elemente von Staatlichkeit finanzieren. Einzelne Elemente von Staatlichkeit, wie die Sicherung des öffentlichen Raumes (z. B. Transportwege), werden von einem Machtpol, andere (wie die Kontrolle und Ausbeutung von Bevölkerung) von einem oder mehreren Machtpolen wahrgenommen. Zwischen ihnen bestehen Beziehungen unter der Bedingung von Anarchie, also ohne eine letztinstanzlich Recht setzende Autorität.

Als Staat wird schon ein Quasi-Staat angenommen, der Entwicklungshilfe erhält und im leicht zugänglichen Gebiet eine meist moderne Armee unterhält, während im „Hinterland" Aufständische oder Drogenbarone mit Hilfe von Guerillakriegern das Gewaltmonopol ausüben und ihren Staatshaushalt aus Rohstoffrenten finanzieren.

Dabei kann durchaus auch der anerkannte Staat die Rente kontrollieren, der subnationale Gewaltakteur dagegen alle übrigen Elemente von Staatlichkeit, z. B. die „Zustim- 
mung“ des „Staatsvolks“. Während des algerischen Bürgerkriegs 1993/96 zog sich der anerkannte Staat aus der Hauptstadt zurück und baute sich eine neue Hauptstadt in einem vorherigen Badeort. Er dirigierte von dort aus von ihm bezahlte Streitkräfte, die vorrangig die Ölfelder, Pipelines und Verladehäfen schützten. Er übertrug der lokalen Bevölkerung die Aufgabe des eigenen Schutzes und wartete, bis das alternative Gesellschaftsprojekt des subnationalen Gewaltakteurs gescheitert war. Dem subnationalen Gewaltakteur war dabei ein beachtliches Maß internationaler Anerkennung zuteil geworden. Er hatte Auslandsvertreter, die formelle Kontakte zu diplomatischen Diensten anderer Staaten knüpften, denen sie mit dem Hinweis auf die de facto-Kontrolle der Bevölkerung und vorübergehend großer Teile des Territoriums nahe legten und versprachen, bei einer bald zu erwartenden Machtübernahme den noch subnationalen Akteur als zukünftigen legitimen Staat anzuerkennen und mit ihm offizielle Beziehungen aufzunehmen bzw. vorzubereiten, um diese Anerkennung als Staat auf den Weg zu bringen.

In dem Maße, wie Renten ohne Mitwirkung einer großen Zahl auf dem Territorium lebender Arbeitskräfte generiert werden können, erlauben Renten, ohne Kontrolle von Staatsvolk und Territorium Elemente von Staatlichkeit aufzubauen. Den Fall, dass dies für den anerkannten Staat zutrifft, haben Beblawi (1987) in Ausweitung der von Mahdawi (1970) und Etienne $(1977,31)$ formulierten Externalität ${ }^{1}$ des Rentierstaats gegenüber der „eigenen“ Gesellschaft dargelegt. Die Theorie der auf Renten gegründeten subnationalen Gewaltakteure behandelt also nur einen Sonderfall: Hier baut der nicht anerkannte und damit subnationale Akteur durch Kontrolle von Renten einen gegen die Bevölkerung und gegen den Staatsapparat gerichteten Gewaltapparat auf.

Soll eine solche Konfiguration der möglichen Trennung zwischen territorialer Kontrolle von Arbeitskraft und territorial eingegrenzter anderer Ressourcen von Macht, z. B. Renten, dauerhaft sein, dann ist zu fragen, weshalb im heutigen internationalen System im Regelfall diese Trennung nicht auftritt und warum ihr nur begrenztes Auftreten historisch ohne Vorläufer ist, obwohl es auch früher territorial eng eingegrenzte Ressourcen gab, deren Kontrolle die Aneignung hoher Einkommen erlaubte: Im vorrevolutionären Frankreich und im tributären China war die Salzsteuer ein solches Instrument, obwohl die Salzlager nicht zu subnationalen Gewaltzentren oder sezessionistischen Tendenzen führten.

Zu klären ist also, weshalb plötzlich im 21. Jahrhundert die Ausübung von Gewalt auf der Grundlage des Zuflusses externer Ressourcen ohne lokale Mitwirkung breiter Teile der Bevölkerung möglich geworden ist.

\section{Der empirische Befund zur These der durch Renten bedingten Zunahme subnationaler Gewalt}

Die „Befriedung“ der kolonial beherrschten Kontinente des Südens seit Ende des 19. bis Mitte des 20. Jahrhunderts war häufig nur „oberflächlich“. Zu Beginn des algerischen Aufstandes 1954 stellte die französische Verwaltung fest, dass es viele Dörfer gab, in denen seit den letzten 15 Jahren kein Vertreter der Kolonialmacht aufgetaucht war. Alle Kolonialgebiete waren chronisch unterverwaltet. Der koloniale Staat war in vieler Hin-

1 Extern, weil er keine Steuern von der Bevölkerung beansprucht und deshalb deren Mitwirkung, aber auch Legitimierung entraten könne. 
sicht abwesend. Erst der postkoloniale Staat erfasste die Bevölkerung jenseits einer auch nur formalen Registrierung zum Zweck einer wie immer willkürlichen Besteuerung. Selbst das alte China kannte breite Gebiete mit nur indirekter Verwaltung und selbst in den Regionen direkter Verwaltung gab es Probleme bei der administrativen Erfassung der Masse der Bevölkerung. Die in der entwicklungsorientierten Verwaltungswissenschaft beklagte Schwäche der kommunalen Selbstverwaltung in der Dritten Welt erweist sich in der neueren ethnologischen Forschung weniger einer zentralstaatlichen Zerstörung lokaler Strukturen geschuldet, denn der Tolerierung der lokalen Selbstorganisation in höchst durchorganisierten Gemeinschaften mit eigenen, oft repressiven Hierarchien auf territorialer, aber vor allem personaler Basis. Das Auftauchen subnationaler Gewaltakteure könnte dann durchaus nur die Sichtbarmachung zuvor existierender Dissidenz sein. Der sich entwickelnde postkoloniale Staat kann sich mit seinen Ressourcen solcher Dissidenz noch nicht unterordnen, muss sie aber wegen seiner größeren „Möglichkeiten“ auch nicht mehr tolerieren.

Die geografische Verteilung subnationaler Gewaltakteure spricht dafür. Als Beleg für die Theorie der auf Renten gegründeten subnationalen Gewaltakteure werden vor allem Systeme aus dem subsaharischen Afrika und dem weiteren karibischen Raum genannt. Sie ließen sich ergänzen durch Randgebiete großer Länder (Indien, Indonesien, Philippinen, Myanmar, Indochina). Subnationale Gewaltakteure sind hier auf Gebiete nationaler oder religiöser Minderheiten beschränkt.

Kennzeichen wie Schattenökonomie oder lokale bewaffnete Einheiten können allerdings durchaus als normale Wirtschaft und regionale Autonomie betrachtet werden. Eine Schattenökonomie entzieht sich dem regulierenden Anspruch eines allogenen Staats, der nach fremden Regeln funktionieren kann, weil er wesentliche Elemente seiner Macht von Ressourcen außerhalb der jeweiligen Gesellschaft bezieht. Der koloniale Staat ähnelt hier dem auf einer im Export angeeigneten Rente aufgebauten Staat. Lokale bewaffnete Kräfte widersprechen dem Prinzip des Gewaltmonopols nur insoweit, dass offenbar zwischen regionaler/lokaler Autonomie und nationalstaatlichem Zentrum kein Ausgleich der Mitwirkungsmöglichkeiten gefunden worden ist.

Wenige Staatswesen des Südens beruhen auf einer durch Zustimmung gegründeten politischen Gemeinschaft. Manche sind Erben tributärer Reiche mit einem nicht immer bis tief in die Gesellschaft reichenden Gemeinschaftsgefühl. Im Begriff des ,nationbuilding" wird thematisiert, dass sich die alten politischen Gemeinschaften, auch Staaten, mit den nachkolonialen Territorialstrukturen nicht decken, die sich durch die Entkolonisierung zu Beginn des 19. Jahrhunderts in Lateinamerika, ab 1930 und verstärkt ab 1945 in Asien und Afrika bildeten.

Gleichwohl ist im größten Teil der entstehenden „Reiche“ ein im Vergleich zu den europäischen Staatsbildungsprozessen relativ niedriges Niveau der gewaltsamen Auseinandersetzung zwischen subnationalen Akteuren und staatlichen Zentren zu beobachten, auch wenn das Eindringen des postkolonialen Staats heute sehr viel tiefer ist als das des kolonialen und des vorkolonialen. Vor allem sind die Systeme mit großen externen Renten nicht durch besonders hohe Niveaus der Aktivität subnationaler Gewaltakteure gekennzeichnet. Aus Libyen, Bahrein, Abu Dhabi, Venezuela und Ecuador wird insgesamt von wenigen subnationale Gewaltakteuren berichtet, die sich Renten aneigneten. Ähnliches gilt für Kupferländer wie Chile und Sambia oder Bauxitländer wie Jamaica und Guinea. Bei Diamanten stehen Sierra Leone eher gewaltfreie Länder wie Botswana, Namibia und Südafrika gegenüber (Snyder/Bhavnani 2005). Die islamistischen Gewaltakteure in Algerien und die marxistischen Gewaltakteure in Peru lassen sich zumindest nicht in ihren Anfangsphasen aus einem Kampf um Aneignung von Renten zugunsten der 
Privilegien der Gewaltakteure erklären. Was bleibt: eine große Zahl substaatlicher Gewaltakteure in relativ kleinen Staaten Zentralamerikas, des nördlichen Südamerikas und des subsaharischen Afrikas sowie in Randzonen der größeren Staaten Asiens, die sich im Regelfall zumindest anfangs aus identitären Konflikten minoritärer Gemeinschaften mit einer auf Zentralisierung drängenden Staatsgewalt entwickeln.

In einer Zone offenbar schwach entwickelter Staatlichkeit kann ein international anerkannter Staat gegenüber der Masse der auf seinem Territorium lebenden Arbeitskräfte seinen Anspruch auf Regulierung und Zugang zu Ressourcen (Steuern) nicht durchsetzen. Gleichwohl ist er aber schon oder noch mächtig genug, den Anspruch rivalisierender Akteure auf solche Kontrolle zu bestreiten. Unter den Bedingungen des heutigen internationalen Systems sind diese Akteure offenbar ausreichend stark, nicht nur auf lokaler Ebene Gehorsam zu verweigern, sondern diese Verweigerung auch gegenüber Gebietsfremden, z. B. mit dem Mittel der Schaffung von nach außen sichtbarer Unsicherheit, zu demonstrieren.

Offenbar haben solche subnationalen Akteure heute ein neues Interesse, mit gebietsfremden Mitgliedern der ,,international anerkannten Welt“ (Staaten, Unternehmen, Nichtregierungsorganisationen) Beziehungen aufzunehmen. Frühere subnationale Gewaltakteure wie die nationalen Befreiungsbewegungen konnten von dieser ,internationalen Welt" die Ressource Anerkennung bekommen, indem sie vorgaben, deren Werten verpflichtet zu sein. Weil die ,international anerkannte Welt" mit dem Ende des Kolonialsystems rechnete, lieferte sie durch Beziehungen und Kontakte Prestige, das sich in der lokalen Wahrnehmung der politischen Großwetterlage zugunsten der Aufständischen auswirkte. Heute dürften die NGOs aus Überlebensinteresse die international anerkannten lokalen Regierungen respektieren. Zur tatsächlich existierenden, möglicherweise ausgeschlossenen Zivilgesellschaft, z. B. ethnisch oder religiös orientierter neuer kulturellidentitärer Bewegungen, besteht wenigstens in den mir bekannten Gebieten so gut wie kein Kontakt, übrigens im Unterschied zu einigen von der politisch korrekten Welt wenig geschätzten Staaten des Südens und ihren Regierungen. ${ }^{2}$ Akteure, die sich gegen die politisch korrekte lokal etablierte Welt auflehnen, dürften deshalb unter den heutigen Bedingungen ähnlich wie zur Zeit des Hochimperialismus von der international anerkannten Welt Leistungen nur erhalten, wenn sie diese direkt in bar bezahlen können. Subnationale Gewaltakteure sind nur dann international sichtbar, wenn sie über Güter verfügen, die sie

2 Das Urteil mag hart erscheinen. Bei unseren Feldforschungen im Rahmen des Projektes „Neue kulturellidentitäre Bewegungen in verschiedenen kulturellen Kontexten“ (Algerien, Indien) bestanden nicht nur zwischen den westlichen Nichtregierungsorganisationen und der realen Zivilgesellschaft keine Kontakte, sondern solche Kontakte wurden auch von beiden Seiten abgelehnt. Das Wissen aller westlichen Organisationen über die reale Zivilgesellschaft war dürftig. Wirklichkeitsnahe Beschreibungen dieses Verhältnisses finden sich in Rieger (2003) und Goonatilake (2006). Die deutsche Politikwissenschaft trägt hier wenig zur Verbesserung bei. Noch vor 10 Jahren schlug eine angesehene westdeutsche Universität auf Platz 1 einer Südasien gewidmeten Professur einen Kandidaten vor, der offensichtlich nur englischsprachige Quellen verarbeitete. Ähnliches scheint für die Drittmittelförderung zu gelten. Über die Selbstverwaltungspraxis der Wissenschaft lässt sich dies nicht ändern, solange der weit überwiegende Teil der deutschen Internationalen Beziehungen das Fach auf den Bereich der Europäischen Einigung und der transatlantischen Beziehungen einschränkt, weil er in der Herausbildung europäischer Identität konstruktivistische Argumente bestätigt sieht. Meine Warnung vor einer weltpolitischen Rolle Deutschlands bleibt deshalb aktuell (Elsenhans 1999). Die geringe Vertrautheit der Protagonisten der Kontroverse über den Kampf der Kulturen mit dem nichteuropäischen Feld ist in der Debatte selten thematisiert worden, also offenbar gar nicht aufgefallen. 
mit guten Erlösen an das politisch korrekte internationale System verkaufen können. Hier sind z. B. die Aktivitäten der Naxaliten in Teilen des indischen Dekkan zu nennen.

Wer von solchen Organisationen auf westliche Hilfe angewiesen ist, weil er keine andere Ressource gegen den übermächtigen international anerkannten Staat einsetzen kann, muss sich auf ein Gewaltverbot einlassen. Die im Kautskyschen Sinn ultraimperialistische Struktur (Kautsky 1914, 921) des anerkannten internationalen Systems verbietet die Unterstützung von Guerillas wenigstens seit dem Zusammenbruch der UdSSR (und damit seit dem Ende der amerikanischen Unterstützung für die Aufständischen in Afghanistan). Wer dennoch bewaffneten Widerstand leistet und einen revolutionären Volkskrieg führt, z. B. die Maoisten in Nepal oder die Kämpfer für ein freies Gurkhaland in Nordwestbengalen, sucht den Kontakt zum international anerkannten politisch korrekten System im Regelfall gar nicht und wird deshalb als Gewaltakteur erst kurz vor der Machtübernahme wahrgenommen, wenn der Übergang von der Guerillataktik zum Kampf mit regulären Verbänden gelungen ist. Wer wahrgenommen wird, sucht vom internationalen System das Einfluss und Hilfe nur noch begrenzt und selten gegen seine lokalen Mitglieder, die anerkannten Regierungen, bereitstellt - Leistungen, die er dann bezahlen muss. Diese Zahlungsfähigkeit ist dann in besonderer Weise gegeben, wenn sich ein solcher subnationaler Akteur einer Rente bemächtigen kann, entweder durch Kontrolle einer schon bestehenden rentengenerierenden Produktion oder durch Aufbau einer solchen Produktion. Es wäre abwegig, Bauern im Aufstandsgebiet zur Produktion besteuerbarer Feldfrüchte anzuhalten, ohne dabei die sehr viel höheren Erlöse für Drogen anstelle der Preise für Nahrungsmittel zu beachten.

Die Rente schafft dann nicht die subnationalen Gewaltakteure, sondern verschafft ihnen auf einfache Weise finanzielle Mittel, die sie sich aber auch in anderer Form mit anderen Mitteln der Ausbeutung so lange beschaffen würden, wie das soziale Problem, auf das sie aufmerksam machen und gegen das sie sich wehren, besteht.

Es bleibt die Behauptung, dass im Unterschied zur Zeit der Entkolonisierung die derzeitigen subnationalen Gewaltakteure keine lokale Massenunterstützung genössen. Unterstützung werde nur durch Terror erlangt. Das Argument war üblich gegen die antikolonialistischen Befreiungsbewegungen. Tatsächlich haben sich alle bewaffneten Widerstandsbewegungen dem Schwinden anfänglich selbst enthusiastischer Unterstützung nicht entziehen können, weil die drückenden Lasten des Krieges allmählich schwerer als die Versprechungen des Erfolges erscheinen. Seit dem Algerienkrieg (1954-1962) setzt die konterrevolutionäre Kriegsführung explizit auf diesen Effekt. Die algerischen Militärs (ab 1992) waren im Bürgerkrieg gegen die Islamisten mit diesem Kalkül erfolgreich. Dieser Ermüdungseffekt wird um so bedeutsamer, je stärker die Erwartungen auf Erfolg der Aufständischen durch Veränderungen des internationalen Klimas und die Übertragung der Stabilisierung westlichen Einflusses auf lokale Staatsklassen schwinden. Die heute anerkannten Regierungen können sich im Regelfall darauf verlassen, dass sie von außen gestützt werden. Nur wenige Besatzer sind so ungeschickt, dass sie diesen Effekt nicht nutzen. Die Kräfte des Widerstands schätzen diese Situation realistisch ein. Die politischen Köpfe sind in den bewaffneten Aufstandsorganisationen im Vergleich zur Zeit der Entkolonisierung schwach geworden, die Romantiker der bewaffneten Aktion dagegen stark, auch weil Veränderungen gegen einen „Ultraimperialismus“ des Nordens nicht durchsetzbar erscheinen (Elsenhans 2002, 208). Dann wäre eine wachsende Gewalttätigkeit Ausdruck einer fehlenden gesellschaftlichen Integration der Widerstandspotentiale in die internationale Gesellschaft und Zeichen der Ausweglosigkeit für Forderungen nach (wie immer auch falsch konzipiertem) gesellschaftlichem Wandel und die Rente finanzielle Basis für die Aufrechterhaltung eines sonst zusammenbrechenden Widerstands bei 
Fortdauer der Anomie. Die Frage der Anomie wäre dann das eigentliche Problem (Zinecker 2006).

\section{Subnationale Gewaltakteure als Folge scheiternden Managements von Renten}

In den größten Regionen der unterentwickelten Welt sind Armut und Elend, aber auch Renten ähnlich verbreitet wie in den Regionen der subnationalen Gewaltakteure. Warum gibt es dann subnationale Gewaltakteure nur in den Randgebieten dieser Kernregionen?

Offenbar waren viele Staatsklassen des Südens auf der Grundlage der Rente durchaus in der Lage, neue Sozialisationsstrukturen aufzubauen, die die Kontrolle der Bevölkerung mit mehr oder weniger spontaner Zustimmung erlaubten. Smith (2004) hat dies für die Ölstaaten aufgezeigt. Oligarchien lateinamerikanischen Typs fehlte diese gewisse Kohärenz in der Nutzung von Renten für den Aufbau tief in die Gesellschaft hineinreichender Klientel- und Patronagestrukturen. Die Staatsklassen der alten tributären Reiche hingen, trotz unterschiedlich starker Bürokratisierung an der Spitze des Integrationsapparats, an der Basis von Personalverbänden ab, deren Bindungswirkung durch verstärkte Marktbeziehungen und daraus wachsenden Optionen der Individuen abnimmt (Familie, Klan, Kaste). Offenbar gelang es vielen der neuen Staatsklassen, eine diese „Atomisierung“ konterkarierende Struktur aufzubauen. In den zwar begrenzten, aber realen Erfolgen ineffizienter Industrialisierung mögen solche Mechanismen liegen. Ähnlich bedürfen neue Dienstleistungen, wie sie auch von den neuen kulturell-identitären Bewegungen bereitgestellt werden, weiterer empirischer Analyse, nicht nur im Hinblick auf die materiellen Ergebnisse, sondern auch im Hinblick auf Erwartungen und Repräsentationsformen von Gesellschaft (Rieger 2003). Auffällig ist die Integrationsfähigkeit jener Systeme, die auf eine vorkapitalistische Tradition von Staatsklassen aus tributären Produktionsweisen zurückgreifen können. Die subnationalen Gewaltakteure treten besonders in Gebieten ohne vorkapitalistische oder vorkoloniale Staatsklassen und geringeren Erfolgen der importsubstituierenden Industrialisierung auf.

Die subnationalen Gewaltakteure wären dann nicht Folge von Renten, sondern der Unfähigkeit der Akteure, Renten politisch so zu nutzen, dass systemstabilisierende Brückenköpfe selbst in einer ansonsten weiterhin dominierten Gesellschaft entstehen.

Die Interpretation des Auftauchens subnationaler Gewaltakteure aus einem Defizit an „capture“ der Rente stimmt mit meiner Analyse der Tendenzen des internationalen Systems überein. Länder, die mit Hilfe des Exports von verarbeiteten Produkten aufholen, beschleunigen den Prozess der Diversifizierung durch „upgrading“, während führende Länder mit aus Renten finanzierten industriepolitischen Programmen technische Führungspositionen in Wachstumsindustrien zu verteidigen suchen. Die daraus resultierenden finanziellen Belastungen werden allenthalben zur Rechtfertigung von Konsumbegrenzungen und Reallohnblockierungen genutzt. Die sich etablierenden politisch administrativen Apparate betreiben im Norden und Süden Rentenaneignung, so dass die Erwartung der Durchsetzung staatsfreier Marktregulierung im globalen System eine (einfältige) Illusion ist (Elsenhans 2009, 39-44). Globalisierung von Rente und nicht Globalisierung von Profit ist angesagt (Elsenhans 2004).

Weil große Wirtschaftsgebiete bei der Umlegung von Startkosten überlegen sind (Hilferding 1910, 446-451), kommt es beim internationalen Wirtschaftssystem zu Isomorphien mit dem sich herausbildenden Großmächtesystem (Multipolarität durch neu aufsteigende Mächte). In einem vermachteten internationalen System unter Dominanz 
saturierter Mächte, die eine Kombination zwischen liberalem (kapitalistischem) Frieden und wohlwollend integrativer Rentenaneignung verfolgen, wird Stabilität gradualistisch und korporativ angestrebt, vornehmlich durch Stützung anerkannter Staatlichkeit gegen subnationale Gewaltakteure, die dann tatsächlich ausgegrenzt sind.

\section{Metatheoretische Überlegungen}

Ist Rente nicht Quelle von subnationaler oder diffuser Gewalt, sondern Element von Stabilisierung in Gesellschaften, in denen unvollständige Durchkapitalisierung das „Empowerment" von Arbeit bremst? Zu diesem Widerspruch der Interpretation kommt es wegen des Problems der Artikulation zwischen ökonomischen und politischen Strukturen in einem Großteil des Mainstreams. Politische Ökonomie glauben heute schon die zu betreiben, die vage davon gehört haben, ökonomische Probleme könnten für das politische Verhalten bedeutsam sein. Meist wird dabei unreflektiert ein neoklassisches Modell des Wirtschaftsprozesses unterstellt (Elsenhans 2000, 568-570).

Tatsächlich beschäftigt sich politische Ökonomie mit den Strukturen, die Akteure schaffen, den Anreizen, die sich aus solchen Strukturen ergeben, und den Eingriffen, die die Akteure aufgrund dieser Anreize wieder in den Strukturen vornehmen, die durchaus auch andere als die intendierten Folgen haben können. In der Politik, Wirtschaft und Gesellschaft umgreifenden Vermittlung von Teilsystemen sind die Akteure nicht stets gezwungen, die finanziellen Ressourcen zu vermehren. Einzelne Akteure können ohne Schaden auch bei den Finanzen nicht finanzielle Zielsetzungen anstreben. Die Ökonomie bestimmt nur in letzter Instanz politisches und gesellschaftliches Verhalten. Gerade weil der Zugang zu Renten nicht vom wirtschaftlichen Wettbewerb abhängt, können Akteure mit solchem Zugang z. B. Ziele der politischen Stabilität verfolgen. Hier gibt es, anders als beim Wettbewerb auf Märkten um kurzfristigen Profit, keine sofort sichtbare Sanktion. Wo diese fehlt, wie bei den Entscheidungsprozessen von Staatsklassen über Investitionen, hängt die Wahl eines Verhaltensmusters von Erwartungen ab. Sie spiegeln empirisch nicht endgültig überprüfte Einschätzungen wider. Die historisch ererbte, konstruierte Geschichte und Gesellschaftsinterpretation interagiert mit dem Lernen aus neuen politischen Herausforderungen, wie dies Zinecker am Beispiel mittelamerikanischer Systeme zeigt (Zinecker 2007, 988 ff.). Geschichte kann dabei in einer Krisensituation offen werden und ist in anderen Situationen pfadabhängig blockiert. Politische Ökonomie beschäftigt sich mit solchen Konfigurationen auf der Grundlage des globalen Vergleiches und der historischen Analyse und arbeitet mit Begriffen wie Produktionsweise und Gesellschaftsformation. Sie wendet sich damit gegen die neuere Entwicklung in der Wirtschaftswissenschaft, in der theoretische Modelle zunehmend den Modellen der Meteorologie ähneln, die begrenzte Regelmäßigkeiten im Chaos systematisieren. Insofern Menschen auch dezentral intentional handeln, schaffen sie über die Projektion eigener Rationalität und die unterstellte Rationalität ihrer Rivalen Verhaltensstetigkeiten, die Modellierungen erlauben.

Zweifellos könnte hier der Konstruktivismus einen Beitrag leisten. Zineckers Theorie des situativ unterschiedlich durchsetzbaren Lernens verweist ausdrücklich darauf. Gerade weil die Akteure bei der Verwendung von Renten relativ frei von ökonomischen Zwängen handeln, ist hier die politologische und soziologische Erforschung der Bildung von Präferenzen und der Weltsichten, in die diese eingebettet sind, wichtig. Es geht dabei aber um eine empirische Erforschung und nicht die plakative Behauptung, konstruktivistische Ansätze seien zur Bekämpfung der realistischen Positionen in der IB-Theorie relevant. 
Der deutsche IB-Konstruktivismus ignoriert die gesellschaftstheoretischen Grundlagen eines Luckmann (Berger/Luckmann 1967) oder Lukács (1967) weitgehend und hat empirisch noch keine größere Arbeit über die Konstruktion von Identität vorgelegt, sondern sich auf die empirische Begründung der Relevanz dieses Bereiches beschränkt (Becker/Müller/Wisotzki 2008) - eine außerhalb der Internationalen Beziehungen völlig unkontroverse Behauptung. Die breite Literatur zu den innenpolitischen Grundlagen außenpolitischen Verhaltens (Ziebura/Ansprenger/Kirsch 1974), die gesamte Kontroverse über den „Primat der Innenpolitik“ (Kehr 1970), so wundervolle Analysen wie die unterschiedliche Ausrichtung des westeuropäischen Imperialismus im Verhältnis zum deutschen (Kehr 1930, 423-425) sind offenbar unbekannt, weil man sich als theoretischer Kontrahent einer im Verhältnis zu den europäischen Realisten des 19. Jahrhunderts einfältigen Rezeption des Realismus entgegenstellt. Internationale Beziehungen und mit ihr die Friedensforschung bleiben weiterhin von den theoretischen Fragen, welche die Sozialwissenschaft zusammenhalten, relativ isoliert.

\section{Bibliographie}

Beblawi, H. (1987) The Rentier State in the Arab World. In: Beblawi, H./Luciani, G. (Ed.) The Rentier State. London: Croom Helm, 49-62.

Becker, U./Müller, H./Wisotzki, S. (2008) Democracy and Nuclear Arms Control. Destiny or Ambiguity. In: Security Studies, 17, 4, 810-854.

Berger, P. L./Luckmann, T. (1967) The Social Construction of Reality: A Treatise in the Sociology of Knowledge. Garden City, New York: Anchor Books.

Bertram, G. W. (1963) Economic Growth and Canadian Industry, 1870-1915: The Staple Model and the Take-Off Hypothesis. In: Canadian Journal of Economic and Political Science, 29, 2 (Mai), 159-184.

Bortkiewicz, L. von (1907) Wertrechnung und Preisrechnung im Marxschen System, 3. Teil. In: Archiv für Sozialwissenschaft und Sozialpolitik, 25, 2, 445-489.

Bulte, E. H./Damania, R./Deacon, R. T. (2005) Resource Intensity, Institutions and Development. In: World Development, 33, 7 (Juli), 1029-1044.

Chateaubriand, F. R. (1981) La Monarchie selon la Charte [1816]. In: Chateaubriand, F. R.: Oeuvres complètes 25. Paris: Lavocat, 3-411.

Chojnacki, S. (2005) Gewaltakteure und Gewaltmärkte. Wandel der Kriegsformen? In: Frech, S./Trummer, P. I. (Ed.) Neue Kriege. Akteure, Gewaltmärkte, Ökonomie. Schwalbach/Ts.: Wochenschau Verlag, 73-100.

Collier, P. (2000) Rebellion as a Quasi-Criminal Activity. In: Journal of Conflict Resolution, 44, 6 (Dezember), 839-853.

Collier, P./Hoeffler, A. (2002) On the Incidence of Civil War in Africa. In: Journal of Conflict Resolution, 46, 1 (Februar), 13-28.

Collier, P./Hoeffler, A. (2005) Resource Rents, Governance and Conflict. In: Journal of Conflict Resolution, 49, 4 (August), 625-633.

Collier, P./Hoeffler, A. Greed and Grievance in Civil War. http://www.worldbank.org/research/conflict/papers/greedgrievance_23oct.pdf $(31 / 08 / 2006)$

Cramer, C. (2002) Homo Economicus Goes to War: Methodological Individualism, Rational Choice and the Political Economy of War. In: World Development, 30, 11 (November), $1845-1864$. 
Dunning, T. (2005) Resource Dependency, Economic Performance and Political Stability. In: Journal of Conflict Resolution, 49, 4 (August), 451-482.

Elsenhans, H. (1994) Rent, State and the Market: The Political Economy of the Transition to Self-sustained Capitalism. In: Pakistan Development Review, 33, 4 (Dezember), 393-428.

Elsenhans, H. (1995) Überwindung von Marginalität als Gegenstand der Armutsbekämpfung. In: Schäfer, H. B. (Ed.) Bevölkerungsdynamik und Grundbedürfnisse in Entwicklungsländern. Schriften des Vereins für Socialpolitik 246. Berlin: Duncker und Humblot, 193-221.

Elsenhans, H. (1999) Reif für die Weltpolitik? Gedanken zur außenpolitischen Elite Deutschlands. In: WeltTrends, 7, 25 (Winter),121-128.

Elsenhans, H. (2000) Political Economy or Economic Politics? The Prospects of Civil Society in an Era of Globalization. In: Indian Journal of Public Administration, 46, 4 (Oktober-Dezember), 567-600.

Elsenhans, H. (2002) Das internationale System seit dem September 2001: Ultrastabilität - Ultraimperialismus - Friedensfähigkeit ohne Entwicklung? In: Asien - Afrika - Lateinamerika, 30, 2 (Juni), 193-218.

Elsenhans, H. (2004) Globalisierung von Profit oder Globalisierung von Rente. In: Jahrbuch für Wirtschaftswissenschaften, 55, 3, 263-289.

Elsenhans, H. (2006) Globalization between A Convoy Model and An Underconsumptionist Threat. Münster: LIT Verlag.

Elsenhans, H. (2009) Kapitalismus kontrovers. Zerklüftung im nicht so sehr kapitalistischen Weltsystem. WeltTrends Papiere 9. Potsdam: Universitätsverlag.

Enders, K./Herberg, H. (1983) The Dutch Disease: Causes, Consequences, Cures and Calmatives. In: Weltwirtschaftliches Archiv, 119, 3, 473-495.

Etienne, B. (1977) L'Algérie, Cultures et Révolution. Paris: Seuil.

Fearon, J. D. (2005) Primary Commodity Exports and Civil War. In: Journal of Conflict Resolution, 49, 4 (August), 483-507.

Ferber, C. v. (1966) Der Werturteilstreit 1909/1959. Versuch einer wissenschaftsgeschichtlichen Interpretation. In: Topitsch, E. (Ed.) Logik der Sozialwissenschaften. Köln: Kiepenheuer \& Witsch, 165-180.

Goonatilake, S. (2006) Recolonisation: Foreign Funded NGOs in Sri Lanka. Beverly Hills; Sage).

Hilferding, R. (1910) Das Finanzkapital. Eine Studie über die jüngste Entwicklung des Kapitalismus. Wien: Brand.

Hugon, P. (2003) Les conflits armés en Afrique : Apports, mythes et limites de l'analyse économique : Entendre les violences. In: Tiers Monde, 44, 176, 829-855.

Humphrey, M. (2005) Natural Resources, Conflict, and Conflict Resolution. In: Journal of Conflict Resolution, 49, 4 (August), 508-537.

Jackson, R. H. (1990) Quasi-States: Souvereignty, International Relations, and the Third World. Cambridge: Cambridge University Press.

Jackson, R. H./Rosberg, C. C. (1985) Juridical Statehood in the Crisis of Tropical African States. Paris: IPSA.

Jensen, N.; Wantchekon, L. (2004) Resource Wealth and Political Regimes in Africa. In: Comparative Political Studies, 37, 7 (September), 816-841.

Kaldor, M. (1999) New and Old Wars. Organized Violence in a Global Era. Stanford, Cal.: Stanford University Press. 
Kehr, E. (1930) Schlachtflottenbau und Parteipolitik 1894-1901. Versuch eines Querschnitts durch die innenpolitischen, sozialen und ideologischen Voraussetzungen des deutschen Imperialismus. Berlin: Dr. Emil Ebering.

Kehr, E. (1970) Englandhaß und Weltpolitik. In: Kehr, E. Der Primat der Innenpolitik. Gesammelte Aufsätze zur preußisch-deutschen Sozialgeschichte im 19. und 20. Jahrhundert. Berlin: Walter de Gruyter, 149-175.

Khan, M. H. (2000) Rents, Efficiency and Growth. In: Khan, M. H./Jomo, K. S. (Ed.) Rents, Rent-Seeking and Economic Development. Theory and Evidence in Asia. Cambridge et al.: Cambridge University Press, 21-69.

Krugman, P. R. (1987) Is Free Trade Passé? In: Journal of Economic Perspectives, 1, 2 (Herbst), 131-144.

LeBillon, P. (2001) Angola's Political Economy of War: The Role of Oil and Diamonds, 1975-2000. In: African Affairs, 100, 398 (January), 55-80.

Lenin, W. I. (1977) Imperialismus als höchstes Stadium des Kapitalismus [1916]. In: Lenin, W. I., Werke (22). Berlin, Bonn: Dietz, 189-309.

Lukács, G. (1967) Geschichte und Klassenbewusstsein. Amsterdam: de Munter.

Mahdavy, H. (1970) Patterns and Problems of Economic Development in Rentier States: The Case of Iran. In: Cook, M. A. (Ed.) Studies in the Economic History of the Middle East: From the Rise of Islam to the Present Day. London/New York/Toronto: Oxford University Press, 428-467.

Malešević, S. (2008) The Sociology of New Wars? Assessing the Causes and Objectives of Contemporary Violent Conflicts. In: International Political Sociology, 2, 2 (Juni), 97-112.

Marx, K. (1974) Vorwort zur Kritik der politischen Ökonomie [1859]. In: Marx, K./Engels F. Marx Engels Werke. Band 13. Berlin: Dietz, 4-11.

Matthies, V. (2005) Eine Welt voller Kriege. In: Frech, S./Trummer, P. I. (Ed.) Neue Kriege. Akteure, Gewaltmärkte, Ökonomie. Schwalbach/Ts.: Wochenschau Verlag, 33-55.

Münkler, H. (2002) Die neuen Kriege. Bonn: Bundeszentrale für Politische Bildung.

Regan, P. M. (2005) Greed, Grievance and Mobilization in Civil Wars. In: Journal of Conflict Resolution, 49, 3 (June), 319-336.

Rieger, B. (2003) Rentiers, Patrone und Gemeinschaft: Soziale Sicherung im Libanon. Frankfurt am Main et al.: Lang.

Ron, J. (2005) Paradigm in Distress? Primary Commodities and Civil War. In: Journal of Conflict Resolution, 49, 4 (August).

Sachs, J. D./Warner, A. M. (1999) The Big Push, Natural Resource Booms and Growth. In: Journal of Development Economics, 59, 43-76.

Schaffar, W. (2008) Birma: Gescheiterter Staat oder (neue) Form peripherer Staatlichkeit. In: Journal für Entwicklungspolitik, 24, 2, 33-62.

Sid Ahmed, A. (1988) De la 'staple theory' à la rente petrolière. In: Tiers Monde, 29, 115, 715-812.

Singer, H. W. (1950) U.S. Foreign Investment in Underdeveloped Areas. The Distribution of Gains between Investing and Borrowing Countries. In: American Economic Review, 40, 2 (May), 473-485.

Smith, B. (2004) Oil Wealth and Regime Survival in the Developing World, 1960-1999. In: American Journal of Political Science, 48, 2, (May), 232-246.

Snyder, R./Bhavnani, R. (2005) Diamonds, Blood and Taxes. A Revenue Centered Framework for Explaining Political Order. In: Journal of Conflict Resolution, 49, 4 (August), 563-597. 
Watkins, M. H. (1963) A Staple Theory of Economic Growth. In: Canadian Journal of Economics and Political Studies, 29, 2 (May), 141-158.

Yi, G.-Y. (1988) Staat und Kapitalakkumulation in ostasiatischen Ländern: Ein Vergleich zwischen Korea und Taiwan. Saarbrücken: Breitenbach.

Ziebura, G./Ansprenger, F./Kiersch, G. (1974) Bestimmungsfaktoren der Außenpolitik in der zweiten Hälfte des 20.Jahrhunderts. Forschungsstrategie und -programm eines Sonderforschungsbereichs. Berlin: Fachbereich Politische Wissenschaft.

Zinecker, H. (2007) Kolumbien und El Salvador im longitudinalen Vergleich. Ein kritischer Beitrag zur Transitionsdebatte. Baden Baden: Nomos.

Zinecker, H. (2006) Gewalt im Frieden. Formen und Ursachen der Nachkriegsgewalt in Guatemala. HSFK Report 8/2006. Frankfurt am Main: Hessische Stiftung Friedensund Konfliktforschung.

Hartmut Elsenhans, Prof. em. Universität Leipzig; Gastprofessuren in Dakar, Lissabon, New Delhi und Salzburg.

E-Mail: helsen@rz.uni-leipzig.de 